\title{
Le corps « dentelé » du Christ
}

Jacques Isolery

\section{(2) OpenEdition}

\section{Journals}

Édition électronique

URL : http://journals.openedition.org/ccs/1030

DOI : $10.4000 /$ ccs. 1030

ISSN : 2558-782X

\section{Éditeur :}

Presses universitaires de Rennes, Association des lecteurs de Claude Simon

\section{Édition imprimée}

Date de publication : 1 décembre 2017

Pagination : 103-112

ISBN : 978-2-7535-5482-5

ISSN : 1774-9425

Référence électronique

Jacques Isolery, «Le corps « dentelé » du Christ », Cahiers Claude Simon [En ligne], 12 | 2017, mis en ligne le 07 décembre 2018, consulté le 14 février 2020. URL : http://journals.openedition.org/ccs/1030 ; DOI : 10.4000/ccs.1030 


\title{
LE CORPS « DENTELÉ » DU CHRIST
}

\author{
Jacques ISOLERY
}

Université de Corse

L'iconographie (Photographies, Album d'un amateur) et les textes de Simon accordent une place non négligeable à la figure du Christ. Pour l'orphelin élevé par une mère profondément croyante dans une région très marquée par les formes spectaculaires de dévotion populaire héritées de la Contre-Réforme et pour l'ancien élève du collège Stanislas, cela n'a en soi rien d'étonnant. Bien qu'évoqué de façon périphérique par rapport à d'autres aspects majeurs de la corporéité, le corps du Christ constitue cependant un corpus non négligeable de souvenirs sensibles, de textes, d'images, de signes, de symboles dont les représentations évoluent dans l'œuvre. Mis tout d'abord en scène dans les dramatisations du baroque populaire et l'évocation du Saint-Sacrement, il apparaît à partir des Géorgiques et dans L'Invitation à travers la mièvre symbolique saint-sulpicienne, pour s'achever dans le thème de la Nativité et de l'adoration des bergers évoquées dans Le Tramway ${ }^{1}$. Même vidé de toute adhésion religieuse de la part de Simon, ce corpus n'en demeure pas moins le support d'une interrogation sur l'incarnation du sens dans la chair des mots et le corps du texte. Aussi, le corps du Christ est-il chez Simon, un triple corps de dramatisation: corps de passion, corps de transfiguration et corps de transgression.

Dans l'histoire de l'Occident, «la foi et la dévotion au corps du Christ ont contribué à élever le corps à une haute dignité ${ }^{2}$ " symbolique et esthétique, puisque ce corps est l'incarnation du Dieu. Parallèlement, elles ont

1. Voir J. Isolery, article "Christ», dans M. Bertrand (dir.), Dictionnaire Claude Simon, Champion, 2013, vol. 1, p. 188-191.

2. J. Gélis, « Le corps, l'église et le sacré » dans G. Vigarelle (dir.), Histoire du corps: de la Renaissance aux Lumières, vol. 1, Le Seuil, coll. «Points Histoire», 2005, p. 17. 
jeté sur la chair et ses tentations le puissant discrédit de la diabolisation des passions. En revanche, le $\mathrm{xx}^{\mathrm{e}}$ siècle, ses traumatismes et atrocités, la marchandisation et la médiatisation des corps mais aussi leur libération ont manifesté l'arbitraire de cette distinction entre corps, chair et esprit. Sciences physiques, psychanalyse ont aussi remis en question l'opposition dualiste newtonienne et cartésienne entre la mécanique des corps et la spiritualité de l'être. La chair est devenue le lieu d'inscription et de médiation entre la complication du corps-machine et la complexité des devenirs du corps organique et des rapports de réciprocité avec son milieu. Aussi bien, si le corps du Christ se trouve dépouillé chez Simon de sa dimension religieuse originelle, il n'en demeure pas moins un des lieux paradigmatiques de la mise en question de tout dualisme ontologique concernant les rapports de la représentation avec la vérité. S'il s'incarne particulièrement dans le registre spectaculaire du corps crucifié et celui, métaphorique, du Saint-Sacrement, c'est que le corps supplicié, l'hostie et la liturgie composent un drame puissamment sensoriel fait de symboles, de rituels et de mots qui s'articulent à la souffrance, à l'angoisse, à la dérision, à la mémoire, aux fantasmes et aux pulsions. Pour cet "obstiné matérialiste ${ }^{3}$ " qu'est Simon, le corps du Christ persiste ainsi à interroger "l'idée de la mort » $(G, \mathrm{p}$. 791), à s'articuler à ses représentations fantasmatiques. Mais surtout, comme l'a souligné Kojève ${ }^{4}$, le dogme spécifiquement et authentiquement chrétien de l'Incarnation a permis de penser que l'idéal peut se faire chair, autrement dit qu'il est possible de résorber les solutions de continuité entre le grand ordre de la Nécessité transcendante et l'apparent désordre hasardeux de l'ici-bas. Or, parmi les médiations possibles entre l'idéal et le matériel, celle du schème joue le rôle de mystérieux intermédiaire entre concept ou catégories, mots et représentations sensibles. L'Incarnation se laisse dès lors interroger comme le paradigme fondateur de cette médiation vis-à-vis de laquelle le texte simonien

3. C. Simon, lettre à Alice Mathiot du 19 janvier 1978, citée dans M. Calle-Gruber, Claude Simon, une vie à écrire, Le Seuil, coll. « Biographie ", 2011, p. 45.

4. «En effet, qu'est-ce l'Incarnation, sinon la possibilité pour le Dieu éternel d'être réellement présent dans le monde temporel où nous vivons nous-mêmes, sans déchoir pour autant de son absolue perfection? Mais si la présence dans le monde sensible ne détériore pas cette perfection, c'est que ce monde est (ou a été, ou sera) lui-même parfait, du moins dans une certaine mesure (mesure que rien n'empêche, d'ailleurs, d'établir avec précision). Si, comme les chrétiens croyants l'affirment, un corps terrestre (humain) peut être "en même temps" le corps de Dieu et donc un corps divin et si, comme le pensaient les savants Grecs, les corps divins (célestes) reflètent correctement des relations éternelles entre des entités mathématiques, rien n'empêche plus de rechercher ces relations dans l'ici-bas autant que dans le ciel. » A. Kojève, L'Origine chrétienne de la science moderne, art. en ligne: [http//etopologie.free.fr/Textes/1_ origine $\% 20$ chretienne $\% 20 \mathrm{de} \% 20 \mathrm{la} \% 20$ science $\% 20$ moderne $\% 20 \mathrm{~A} . \% 20$ Kojeve(revue).htm]. 
semble hésiter, alterner ou jouer entre la résilience d'une fascination primitive et la distanciation critique ironique.

\section{UNE PASSION CONTAGIEUSE}

Le Crucifié apparaît dès Gulliver dans la mise en scène d'un schème agonistique qui répand sa complexion sur les lieux entremêlés de toute passion, que son expression soit religieuse, érotique, guerrière ou herméneutique. Le corps du Christ est un des topoï où s'exprime de façon spectaculaire la cruauté des forces paradoxales de mouvements expansifs et d'immobilisme morbide. Évoquant les pratiques dévotes de la mère de Tom, métisse mexicaine, le narrateur de Gulliver en souligne l'étrangeté et le rayonnement contagieux. La femme s'est installée dans la vieille maison

[s]ans rien ajouter au poussiéreux ameublement qu'un autel aux broderies empesées et immaculées sur lequel trônait un crucifix à demi enfoui dans les fleurs (leur parfum poivré pénétrant, cadavérique, se répandant, prenant possession d'une pièce après l'autre) elle avait peu à peu fait régner dans la maison tout entière cette étrange et macabre atmosphère au sein de laquelle un Dieu supplicié et sans pitié semblait, au milieu des pétales exhalant leur lent poison, le symbole erroné d'un dogme trompeur d'espérance restitué à sa véritable destination, cloué pour l'éternité dans son effroyable agonie parmi la profusion végétale luxuriante, magnifique, d'une vie inexorable et sanguinaire ${ }^{5}$.

Avec ses métaphores, ses oxymores et ses antithèses, ses modalisations, ses effets de submersion sensible et de distanciation critique, cette phrase « cruciale " constitue une mise en abyme programmatique de nombreux thèmes simoniens et réseaux de représentations sensibles ainsi qu'une incarnation sur le plan stylistique et pragmatique de cette esthétique de la contagion que développe son sémantisme dénotatif et connotatif. La vieille maison " au poussiéreux ameublement » oppose, à travers la figure de la métisse, l'ancien et le nouveau. À la fois métaphorique, métonymique et allégorique, l'image évoque implicitement les grandes découvertes et la colonisation espagnole, placées sous le signe sanguinaire de la conversion au Christ que Simon évoquera dans Orion aveugle et Les Corps conducteurs. Mais par un effet de révolution, au sens que Le Palace donne à ce terme, c'est ici le vieux monde que viennent envahir les modes superlatifs et barbares de cette dévotion à la croix où le crucifix joue son rôle d'axis mundi. Simon souligne dans Album d'un amateur les effets de contamination des ondes circulaires de « cette civilisation [...] de la croix reconnaissable au premier coup d'œil » $(P, \mathrm{p} .462)$. 
Et si « [p]ar le Mexique, l'Espagne a exporté ses traditions de dévotion ${ }^{6}$ ", celles-ci se sont teintées, souligne Jean-Yves Laurichesse, "des fastes cruels de la religion aztèque ${ }^{7}$ ", pour faire retour au vieux continent ${ }^{8}$. La figure du Crucifié dresse ainsi chez Simon un carrefour entre civilisation et barbarie, raffinement et brutalité, vie et mort, richesse et pauvreté, durée et éternité, sublime et trivialité. Elle est un des lieux de l'opposition oxymorique et de sa résolution dans le texte.

Le lecteur simonien décrypte aisément sous cette longue phrase, la préfiguration de toutes les séries narratives ultérieurement soumises au double phénomène d'insularisation et de contamination. Tout lieu fermé comme un "îlot» $(A, \mathrm{p} .1136)$, en cherchant à se préserver de l'extérieur, finit par fabriquer ses propres mouvements d'intussusception morbide et de contagion. Ce phénomène concerne tout autant la matrice maternelle, que la chambre et les vieilles maisons de Perpignan ou du Jura, les caves et caveaux, le bureau d'oncle Charles, le collège Stanislas (JP, p. 1038-1039), les photos et cartes postales, etc. Tout espace confiné tend d'un côté à l'immobilité entropique et ne cesse, de l'autre, d'activer malgré lui les mouvements d'un imaginaire par lequel le lieu redevient un milieu au sein duquel s'opèrent des alchimies, des mélanges et des contaminations expansives. Ni l'insularité stylistique de la période ni l'archipel de ses clauses, selon la terminologie proposée par David Zemmour, n'échappent à ces phénomènes d'amplification et d'épanchement métaphoro-métonymiques de cette syntaxe $d u$ sensible ${ }^{9}$. Ainsi, le souffle ample de ses nombreuses clauses semble éterniser la citation de Gulliver dans une "lente agonie ", s'achevant sur la résurrection paradoxale in fine de la polysémie du terme "sanguinaire ». Ici aussi, le mimétisme de cette phrase "agonisante " en annonce bien d'autres à venir si typiques de l'écriture simonienne.

6. Alb., p. 46.

7. J.-Y. Laurichesse, La Bataille des odeurs, L'Harmattan, coll. «Littératures », 1998, p. 129.

8. Quitte à s'exercer enfin à New York, "à l'enseigne de cette San Gondolfo Society inc." (Alb., p. 48) dont le saint tutélaire d'origine milanaise et d'obédience franciscaine redonna la parole et l'ouïe à un sourd muet à Castelvetrano, en Sicile, dans les années 1255-60, quelques années avant le massacre des vêpres siciliennes.

9. D. Zemmour, Une syntaxe du sensible: Claude Simon et l'écriture de la perception, PUPS, "Travaux de stylistique et de linguistiques françaises ", 2008. 
Dans la chambre d'agonie de la mère, où se déroule dans Histoire le rituel de la messe, la luxuriance mortifère des fleurs ${ }^{10}$ omniprésentes fixe cette " odeur même du tabou " maternel que Jean-Yves Laurichesse a clairement explicitée ${ }^{11}$. Mais ici, le corps du Christ, remplacé par l'acronyme INRI (Iesus Nazarenus Rex Iudaeorum) inscrit en "caractères épineux eux aussi griffus gothiques et entrelacés INRI " (Hist., p. 152), est relayé par le souvenir de la photographie sépia d'Henri, le père, cette autre « divinité au système pileux bouclé et soyeux » dont le "sourire hardi ironique et indéfectiblement optimiste " (ibid., p. 153) semble vouloir réfuter la tragédie familiale, l'angoisse du temps, l'horreur de la maladie. Ce que restitue l'écriture adulte de cette " inoubliable image flottant immatérielle et auréolée de brouillard " (ibid.) qui participe d'une ambiguïté aussi troublante que le mélange de la rose féminine et mystique, de l'arum phallique et des œillets ou que l'érection de la croix sur l'autel fleuri. La consolation promise par ce Paraclet paternel est intrinsèquement liée à la mort, passage nécessaire pour «le rejoindre [dans] un au-delà paradisiaque et vaguement oriental quelque Eden quelque jardin à l'inimaginable végétation " (ibid.). Le tabou ne cesse d'engendrer de subtiles transgressions que l'écriture suggère sous forme d'analogies implicites, de réseaux associatifs, de figures rhétoriques. L'image récurrente des boucles soyeuses du Christ-père renvoie à d'autres pilosités, aux lingeries féminines - voir les "soyeuses lingeries " de Corinne (RF, p. 207) -, aux végétations et broussailles plus intimes. Le calice s'associe aux seins ou au sexe de la femme dans Le Jardin des Plantes ${ }^{12}$. Quant à l'autel, c'est un des termes qu'emploie Simon pour désigner métaphoriquement le sexe féminin sur lequel viennent se crucifier les passions érotiques et scopiques masculines: œillades suggérées en quelque sorte par les œillets.

Parce qu'il s'y est " crucifié, agonisant sur l'autel la bouche l'antre de... » Corinne ( $R F$, p. 200), le capitaine de Reixach d'abord, Iglésia peut-être, Georges par la suite, ont tous les trois " consommé si l'on peut dire cette Passion, avec cette différence que le lieu le centre l'autel n'en était pas une colline chauve, mais ce suave et tendre et vertigineux et broussailleux et secret repli de la chair..." (ibid.). Or, "la chair du monde est femelle "

10. Les «fleurs peintes" (Hist., p. 151) des vases, les " petits paniers fleuris" (p. 153) du papier peint, "les guirlandes de roses" (p. 152) du tapis et celles de la chasuble du prêtre ornée de "rosiers grimpants s'entrecroisant s'enlaçant dessinant des huits épineux comme des ronces " (p. 151) et enfin les roses et arums (p. 407).

11. J.-Y. Laurichesse, op. cit., p. 125.

12. Voir, par exemple, JP, p. $926-927$ et p. 967. 
( $V$, p. 75), elle est cette " palpitation secrète, multiple, mystérieuse » (ibid.) qui détient la marque de l'entre-deux, du " pertuis " (JP, p. 1120), du pli entre le vide et le plein, entre le visible et l'invisible, le dicible et l'indicible, entre l'ontologie énigmatique de l'être féminin matriciel et la logologie de ses devenirs métaphoriques dans la genesis de l'écriture: "moule poulpe pulpe vulve - faisant penser à ces organismes marins et carnivores aveugles mais pourvus de lèvres, de cils" ( $R F$, p. 220). Mais le corps du Christ entretient lui aussi un rapport étroit avec l'œil et la bouche qui trouve son expression privilégiée, non seulement dans les symboles du poisson-phallus, de l'autel-vagin ou de l'hostie-bonbon, mais aussi dans l'évocation récurrente d'une matière sur laquelle il convient de s'attarder: la dentelle.

\section{LE CORPS DENTELÉ DU CHRIST}

Phonétiquement, le mot "dentelle " (ou "petites dents ») contient toutes les violences potentielles du phonème ${ }^{13}$ [dent/dans] accolé à tout ce que peut virtuellement solliciter le phonème [elle/aile]. Ces phonèmes situent la dentelle au carrefour d'un complexe où se rejoignent la bouche et les dents, l'œil, le dehors et le dedans, l'oraison et l'inexorable, la dévoration et la scrutation, la langue et la parole, la matrice et la mort, les rapports souvent violents entre masculin et féminin. Contrastant mais s'associant avec la barbarie des arma Christi - croix, lance, couronne d'épines, clous -, avec « la flamboyante auréole métallique aux pointes acérées qui ressemblent à des poignards " (Alb., p. 48), avec les « somptueuses robes de douleur» (ibid.) des vierges, le fragile raffinement de la dentelle est intimement lié à la barbarie masculine de la statuaire du Crucifié et à certains traits du féminin: «Dans le midi de la France, pendant la Semaine sainte, le corps du Christ revêtu d'une jupe de dentelle empesée est exposé sur des lits de fleurs" (Alb., p. 48). Blanche et immaculée, la dentelle des coussins sur lesquels repose la mère agonisante évoque " une parure de mariée" (Hist., p. 407). Poignardée au cours d'un coït, Nastasia Philippovna gît sous " un fouillis de dentelles blanches" (Alb., p. 14). Mais les huîtres japonaises dessinent aussi "comme une dentelle de pointes reliées entre elles par des membranes de nacre" (JP, p. 1124).

La dentelle permet ainsi d'exploiter un registre binaire très récurrent chez Simon. Sa blancheur ponctue le corps « nu, noir et juif» $(V$, p. 30) du Christ. Immaculée, elle convoque un imaginaire antagoniste: celui du sang, 
de la défloration et des représentations qui donnent corps à "lidée de la mort" ( $G$, p. 791), à son schème. Elle réveille le souvenir des "mises en scène barbares et funèbres" de la Semaine sainte, des éponges imprégnées d'eau de Cologne faites elles aussi "d'une matière dentelée ", $\mathrm{du}$ "col de dentelle blanche" (ibid.) des robes de pénitents ou de prêtres, des baisers apposés sur les pieds du Christ décharné " par les lèvres des amoureuses et des enfants " ( $V$, p. 30), les êtres fragiles par excellence. Sa présence récurrente entretient une ambiguïté d'ordre sensoriel et sexuel. Dans le travelling qui restitue le regard de l'enfant, la dentelle prélude à la vision " des gouttes de sang en relief qui sécoulent de la blessure elle-même comme une bouche aux lèvres gonflées " ( $G$, p. 791) confirmant ce noud entre l'œil et la bouche, entre violence, souffrance et jouissance. D'ailleurs, "n'y avait-il pas aussi une putain làbas » $(R F$, p. 200) ?

Contrairement à la broderie qui augmente l'aspect décoratif d'un support existant ${ }^{14}$, ou au "grossier faufilage qui relie l'innommable au nommé » (Hist., p. 328), la dentelle est réalisée sans trame ni chaîne. Elle consiste en un entrelacement savant de multiples fils entrecroisés ou noués autour d'un vide qu'elle façonne. Dans un registre d'analogie, la dentelle ne peut donc pas être assimilée au tissage de l'écriture qui se déploie sur la chaîne syntagmatique et la trame paradigmatique. Elle renvoie plus explicitement à un processus de lecture des signes. De même que l'arabesque où Stéphanie Orace repère une image de l'intratextualité ${ }^{15}$, la dentelle combine les figures de la boucle, de l'entrelacement, du serpent, du croisement, de la ramification, de l'arborescence. Elle possède, comme l'arabesque, un fort caractère digital: elle fait signe vers un objet possible dont elle masque et fragmente la reconnaissance, faisant de cet objet une différance. Sa texture, faite de pleins façonnant des vides, est telle que l'œil doit choisir entre accommodation sur le motif ou focalisation sur ce qu'il dévoile en cachant. Elle stimule un désir de gestalt et fait obstacle à la réalisation de ce désir, de même que la Loi dans l'Évangile paulinien. En ce sens, la dentelle peut servir d'analogie au choix prétendument drastique que Jean Ricardou posait naguère quant aux deux options de lecture: soit "l'illusion référentielle " $(J P$, p. 1162) qui projette derrière la dentelle du récit ses propres schèmes et fantasmes, soit «l'illusion littérale » qui se laisse «fasciner par la pure matérialité » de la

14. Dans La Route des Flandres, Georges déclare: "lui [Blum] disait que j'inventais brodais sur rien " $(R F$, p. 399). La broderie relève du romanesque narratif, de l'assaisonnement dirait Aristote.

15. S. Orace, article "Arabesque ", dans Dictionnaire Claude Simon, éd. cit., t. 1, p. 55. 
lettre et qui, poussée en ses extrêmes, rejoint alors la poésie sonore et onomatopéique de la langue barbare, cette "dissonance" du poétique contre l'analytique cognitive qu'évoque Jean-Marie Schaeffer ${ }^{16}$. Or, ces deux lectures ne sont pas inconciliables et l'écriture de Simon fait elle aussi lever « ce chant des émotions que la poésie met en scène, en déployant la stratification langagière (sons, rythmes, syntaxe, sémantique, images, etc.), en travaillant les possibilités propres de chacune des strates et en établissant des relations polyphoniques entre elles ", comme l'avait déjà remarqué Roman Ingarden ${ }^{17}$.

\section{LE DIVERS}

Il n'est donc pas si sûr qu'avec le corps dentelé du Christ, nous soyons en présence d'une disjonction exclusive. Le pagne en dentelle qui voile la nudité du Christ questionne son identité sexuelle et son épineuse essence à la fois humaine et divine. Comme le crêpe Georgette ou le brouillard, la dentelle provoque en fait ce qu'llias Yocaris appelle un "effet de "voilé/dévoilé" 18 " où le regard se trouve confronté à un "état "intermédiaire" entre l'être et le non-être, entre la présence et l'absence ${ }^{19}$ ". Dieu peut-il s'incarner en un corps sexué? L'idéal peut-il se faire chair? Le corps et le sang peuvent-ils se transsubstantier dans l'eucharistie? En posant sur le corps du Christ le signe en clair-obscur de sa matérialité, la dentelle n'en laisse transparaître que les fragments d'une chair morcelée, côtoyant la chair cloutée et la chair blessée. Cette chair humaine et trop humaine renvoie alors à l'horizontalité macabre « du Christ mort peint par Holbein [qui] peut faire perdre la foi » (JP, p. 951), selon l'opinion de Dostoïevski transcrite dans Le Jardin des Plantes. Mais déjà, la "pastille blanche " (Hist., p. 150) qu'engloutit la grand-mère "avec une expression crispée de souffrance et de gourmande béatitude " n'est guère moins irrévérencieuse que la "bite à Jésus " (Hist., p. 239) qu'évoque Lambert dans Histoire. L'une assimile l'eucharistie à un "bonbon ", l'autre fait trivialement basculer le corps de Christ dans la nudité et l'organique du corps animal. Mais si ces deux attitudes ont quelque chose de blasphématoire, c'est bien moins vis-à-vis de la dimension trans-

16. J.-M. Schaeffer, Petite écologie des études littéraires, Éditions Thierry Marchaisse, 2011, p. 116.

17. Cité par J.-M. Schaeffer, ibid.

18. I. Yocaris, "Sous le pagne de Jésus", Revue des Lettres Modernes, série "Claude Simon ", n 4 ("Le (dé)goût de l'archive »), Minard, 2005, p. 198.

19. Ibid., p. 196. 
cendantale du religieux qu'à l'égard de ce vertige de l'altérité que Segalen nommait "la perception du divers ${ }^{20}$ ", un vertige qui fait connaître " que quelque chose n'est pas soi-même».

La dentelle permet ainsi de contourner aussi bien l'éventualité d'une foi que Simon ne possède pas que celle du blasphème qui en est une forme d'inversion vaguement sadienne. Elle se contente d'être le signe perceptif d'une question, de dissoudre les distinctions identitaires trop claires, de provoquer ce «mouvement ondulatoire de la conscience ${ }^{21}$ » entre le dehors et le dedans qu'évoque David Zemmour. Si Montès est une figure de l'hostia, de la victime qui « attire les humiliations et les coups ${ }^{22}$ " mais aussi les enfants et les femmes comme l'a souligné Jean-Yves Laurichesse, il ne possède, pas plus que le vent ni que les révolutionnaires espagnols à l'aspect de "christs lilliputiens et consumés» $\left(P\right.$, p. 488), le privilège de l' "oubli ${ }^{23} »(V$, p. 191), homonyme, soit dit en passant, de l'hostie. Le corps dentelé du Christ dresse ainsi un carrefour de questions qui déconcertent non seulement la possibilité mais aussi la nécessité pour une représentation sensible de résoudre le hiatus entre l'abstraction de l'idéal et les formes concrètes du réel empirique. Il est, comme le sexe féminin, le signe productif de l'entre-deux, la métaphore de l'imaginaire, posé comme une écriture blanche sur une réalité qui se refuse en s'offrant, un objet qui s'offre en se refusant ${ }^{24}$. La dentelle serait ainsi une figure de synthèse des potentialités créatrices de l'imagination schématisante, prise entre réel, symbolique et imaginaire, sans cesse dynamisée par «le rythme d'alternance du schéma et de la correction ${ }^{25}$ ».

D'ailleurs, il suffit que la dentelle vieillisse, s' effiloche et se ternisse pour que ses "festons javellisés " $(C B$, p. 559) laissent apparaître cette même chair d'humanité mais sous son angle mercenaire. Chair des putains dans La Chevelure de Bérénice, "leur chair tout entière épaules poitrine cuisses décolorée ", exposée dans un bordel et dépouillée de tout attrait car de tout

20. V. Segalen, Essai sur l'exotisme, Fata Morgana, coll. « Biblio essais», 1978, p. 41.

21. D. Zemmour, op. cit., p. 298.

22. J.-Y. Laurichesse, article "Retable ", dans Dictionnaire Claude Simon, éd. cit., t. 2, p. 912.

23. Dès le $\mathrm{XII}^{\mathrm{e}}$ siècle, l'hostie est une rondelle de pâte cuite entre deux plaques de fer comme une oublie par les oubloyers (ou oublieurs).

24. À ce titre, métaphore possible de la textualité, la dentelle serait une réponse à la sonnette d'alarme tirée lors de la journée d'étude sur le corps (séminaire de l'Association des Lecteurs de Claude Simon du 14 juin 2014) par Pascal Mougin sur le "trop bon fonctionnement du texte simonien " et la menace d'un bouclage, d'un verrouillage interprétatif.

25. E. Gombrich, L'Art et lillusion (1960), tr. G. Durand, Gallimard, 1996, p. 231. 
mystère, celui-ci se déplaçant sur « une guirlande de roses [...] peinte [...] leurs tiges entrelacées épineuses entourant le chiffre huit » (ibid.), tout comme la chasuble du prêtre s'orne dans Histoire de « rosiers grimpants s'entrecroisant s'enlaçant dessinant des huits épineux comme des ronces" (Hist., p. 151). Arabesque... Cette chair exposée, dénudée, dépouillée trouve alors son répondant dans le geste exhibitionniste d' " un Christ aux blondes pilosités écartant d'une main l'ouverture de sa tunique d'un bleu acide " pour montrer du doigt son « cour d'or flamboyant " $(I$, p. 997).

\section{CONCLUSION}

" Il n'y a pas de Mystère dans un monde homogène ${ }^{26}$ ». La dentelle, les floraisons, les corps sont autant de carrefours de l'imaginaire textuel où se rejoignent le fantasme du jardin mystique, les questions d'identité sexuelle, les justifications de la souffrance, de la révolte, de la cruauté ou de la mort. C'est à ce titre que le corps du Christ appartient aux nombreuses représentations d'un schème amphibologique qui n'offre de rédemption que celle offerte par l'expérience phénoménologique du divers et la tentative de reconstitution de cette expérience par une mémoire, certes inquiète, mais dont l'inquiétude - celle de l'auteur comme du lecteur - façonne une esthétique fractale hologigogne ${ }^{27}$, turbulente. Tant du point de vue de l'écriture que de celui de sa réception, ce corps s'inscrit dans une éthique de la différence à la fois créatrice et interprétative contre la menace des schémas trop normatifs. Encore faut-il souligner que la reprise thématique ou stylistique tend, elle aussi, à façonner ses propres schémas « simoniens "... Il n'y a de résurrection chez Simon que celle d'une réalité sensible dans le moment d'écriture et il n'y a de corps signifiant que celui du texte, de son ossature solide, de ses articulations complexes et de sa chair sonore et visuelle. Quant à son sang, c'est au lecteur qu'il revient d'en opérer la perfusion. Ce sens toujours incorporé, cela s'appelle la communion.

26. V. Segalen, Essai sur le Mystérieux, cité dans Marc Gontard, "Victor Segalen: de l'altérité à l'archipélité ", dans G. Voisset (dir.), L'Imaginaire de l'archipel, Karthala, 2003, p. 169.

27. «Les objets fractals peuvent être envisagés comme des structures gigognes en tout point (et pas seulement en un certain nombre de points, les attracteurs de la structure gigogne classique). Cette conception des fractales comme cas particuliers de structures gigognes implique une définition tautologique: un objet fractal est un objet dont chaque point est un objet fractal » (P. Boulanger et A. Cohen, Le Trésor des Paradoxes, Belin, 2007, p. 67). 